\title{
A Comparison of Human Performance between Operators of a Main Control Room in the SMR
}

\author{
Eun Mee Heo ${ }^{1}$, Seong Nam Byun'2, Hong Joon Park ${ }^{3}$, Geun Ok Park \\ ${ }^{1,3}$ Department of Industrial Engineering, The University of Kyunghee, Yong-in, 446-701 \\ ${ }^{2}$ Department of Industrial \& Management, The University of Kyunghee, Yong-in, 446-701 \\ ${ }^{4}$ Korea Atomic Energy Research Institute(KAERI), Daejeon, 305-353
}

\author{
Corresponding Author \\ Seong Nam Byun \\ Department of Industrial \& Management \\ Engineering, The University of Kyunghee, \\ Yong-in, 446-701 \\ Mobile : $+82-10-6339-7081$ \\ Email : snbyun@khu.ac.kr
}

Received : December 11, 2013

Revised : January 27, 2014

Accepted: February 04, 2014
Objective: This study aims to improve human performance by analyzing the operators' tasks and providing input data on the composition of future SMART operators.

Background: SMART is a nuclear reactor for export which needs operators who can satisfy both safety and economic feasibility. Therefore, this study is fundamental research on the composition of operators and this research analyzed SMART tasks in terms of human safety performance.

Method: After analyzing 10 SMART EOG in hierarchical task analysis, this study classified task performance types according to task requirements of NUREG-0711 (Rev.3).

Results: This study found the task frequency of SMART EOG and 12 operating task types.

Conclusion: This study expects that human performance can be improved by analyzing the personal errors, which have the highest task frequency among 12 operating task types.

Application: The results of this study can be applied as base data when licensing needs to be acquired.

Keywords: Task analysis, SMART, Human performance, Team performance

\section{Introduction}

Many incidents and accidents have been experienced in the aviation, railroad, port, airport, chemical, oil refining and medical industries, as well as in the nuclear power industry so far, due to human errors. For this reason, the human error reducing activities draw attention (Campbell, 1971; Goldstein, 1980; Latham, 1988; Salas and Cannon-Bowers, 2001; Tannenbaum and Yukl, 1992; Wexley, 1984). In the past, human errors were approached as just individual problems, and also measures to prevent those errors were regarded as a personal issue. Human errors, however, are approached as an organizational problem, not as an individual problem, and prevention of those human errors is focused at organizational level. In Korea's nuclear power plant MCR, a team comprised of five such members as shift supervisor (SS),

\footnotetext{
Copyright@2014 by Ergonomics Society of Korea. All right reserved.

(1) This is an open-access article distributed under the terms of the Creative Commons Attribution Non-Commercial License (http:// creativecommons.org/licenses/by-nc/3.0/), which permits unrestricted non-commercial use, distribution, and reproduction in any medium, provided the original work is properly cited.
} 
shift technical advisor (STA), reactor operator (RO), turbine operator (TO) and electric operator (EO) is carrying out operation work to achieve common goal of safe power plant operation. APR-1400 (Shingori Units 3\&4) currently preparing for getting the operation permit has been designed as Korean style cutting-edge digital control mode of main control room. The operation team consists of 10 members ( 5 for main control room and 5 for local control stations) in the same manner as the existing control room. The operators of SMART (System-integrated Modular Advanced ReacTor) MCR, which is a small and medium sized reactor $(\mathrm{SMR})$, three team members, comprised of a senior reactor operator (SRO) and an assistant operator (AO), in charge of normal operations under the changed digital environment, and a local operator (LO), in charge of abnormal and emergency operations, carry out operations. This means that team performance may vary according to team characteristics, team competencies and team skills, in addition to adaptation to new technologies of the digital environment. Studies on individual operator's performance and reliability are actively conducted in the nuclear power industry at home and abroad (Carter and Uhrig, 1989; Coblentz, 1988; Rasmussen, Duncan, and Lepplat, 1987; O'Hara and Hall, 1990; Byun and Lee, 2000; etc.). However, there is a limitation with the existing approach on the human errors, as new human errors appear, according to adoption of digital equipment. The reason is that operator's adaptability to new technologies is required, as digital technologies are applied. For human errors on digital equipment, an approach for prior prevention is necessary through experimental and analytical research on the human error mechanism (KAERI/TR-4575, 2011). For cutting-edge digital technology, not only individual operator's knowledge and skills, but technology development to improve human performance by analyzing and evaluating the factors for operators' teamwork and collaboration that can exert higher performance and reliability through organic combination of the whole operation team is important. This study aims to classify task types by operator by analyzing SMART operation tasks to ensure sufficient competitive safety and economic feasibility in comparison with other similar nuclear power plants, as an export-driven small and medium sized nuclear power plant.

\section{Contents of Study}

Most digital-based nuclear power plant entrepreneurs observe the operation team in regards with how many operators should compose an operation team and on whether the number of operators is rational, although it depends on situation. The reduction of one or two operators from the operation team means an important decrease in term of control room resource. Dyer (1984), who pointed out the need for the study on a team, described that the definition of a team cannot be clearly made. He pointed out that a team is specialized for two or more people, common goal and each individual, and thus, includes assigned tasks and interaction. After that, studies on the factors of a team were reestablished by many researchers in the 1980s (i.e., Modrick, 1986; Morgan, Glickman, Woodard, Blaiwes and Salas, 1986). Salas defined that team members perform tasks (or functions) assigned to each of them, and the team has limited lifespan. Orasanu and Salas (1993) pinpointed the factors to be included in a team: First, a team needs to make a decision in huge task performing process. Second, a team member should be equipped with knowledge and skills related with the task concerned and team's decision making. Third, the task may sometimes have high workload and under temporal pressure. The unique characteristics and definitions of a team are the goal-oriented expressions to improve team performance. The team's unique characteristics to improve team performance are defined as follows (Salas, Burke, and Cannon-Bowers, 2000): First, team members are mutually dependent, and their behaviors need to be adjusted efficiently in line with other team members through collaboration. Second, if tasks need to be performed under the complex environment, team members need to dynamically transform information and resources in the same manner as communication. Third, a team has limited lifespan, therefore, team members need to actively work towards common goal through sharing information between them. Team size affects how team members perform tasks and how they behavior between them (Curral, Forrester, Dawson, and West, 2001). The team size, comprised of 8 to 10 team members, is known to be the ideal team size that can maximize team performance. Shaw (1981) asserted 5 to 7 team members are ideal in the case of performing very complicated tasks as shown in the operation work of nuclear power plant's main control room. Meanwhile, Likert (1977) said a team tends to perform tasks through being divided, team task performing processes are not clear, and explicit team goal may lose, if team members are too many, namely over 10. Seaman (1981) insisted that more problems occur in performing tasks in proportion with an increase of 
team size. In the meantime, there is no study on the optimum small team size. Jackson (1996) pointed out lacking diversity of team members may cause some problems in performing tasks with various standpoints, if team members are two or three. NUREG/IA-0137 compared and verified situation awareness, mental workload and team interaction, after assuming the minimum operators of a cutting-edge control room (SRO, RO or Balance of Plant Operator). The result was that the situation awareness and team interaction of cutting-edge control room composed of two people were higher than those of the conventional control room composed of four people (SRO, RO, BOP Operator and Control Room Technician). Cannon-Bowers et al. (1995) asserted that team competencies are dependent on task characteristics and team characteristics. The adjustment and cooperation between team members may vary, according to team competencies, and he defined such team interaction as teamwork. Many researchers sought factors for efficient teamwork (Salas, Sims and Burke, 2004). They presented team leadership, mutual performance monitoring, backup behavior, adaptability/flexibility and team orientation as the essential factors of teamwork. They also emphasized shared mental models, closed-loop communication and mutual trust as three team adjustment mechanisms.

\subsection{Human Performance and Team Performance}

For Human Factors Engineering Verification and Validation(HFE V\&V) on domestic digital-based main control room design, the KSAX (Korea Situational Awareness Index) is used by modifying BARS (Behaviorally Anchored Rating Scales), NASA-TLX (National Aeronautic \& Space Administration-Task Load Index) and SART (Situation Awareness Rating Technique) to be in line with Korea's situation to measure team performance (team interaction), according to each team, and human performance (mental workload and situation awareness).

From the aspect of human performance, NSAA-TLX classified six such factors as mental demand, physical demand, temporal demand, performance, effort and frustration level as the factors affecting workload (Hart and Staveland, 1988). Workload is decided by multiplying weight by evaluated value on each factor through pairwise comparison of those factors.

The situation awareness of a nuclear power plant MCR operator can be defined as sensing various situation information occurring from various marks and control facilities, comparing pre-set operation goal, precisely understanding the meaning of presented situation information, and then predicting how power plant state will change in the future. Situation awareness is highly related with human performance, and thus outstanding situation awareness is connected to high human performance (Endsley, 1995). Human performance is not always bad, even though situation awareness level is low. For example, if one admits his/her situation awareness function is not good, and is careful about decision making and task behaviors, the situation awareness does not greatly affect human performance (Endsley, 1990; Venturino et al., 1989). Especially, situation awareness is one of the standards explaining control task performing worker's behavior, and is widely used as an important concept in the aviation industry field like airplane operation, air control, airplane maintenance/repair, and the large control system operation field including chemical factories and oil refineries, as well as the medial sector (Adams et al., 1995; Endsley and Robertson, 2000; Endsley and Rodgers, 1997; Gaba et al., 1995; Hallbert, 1997; Itoch and Inagaki, 1996; Kaber and Endsley, 1998; Sandom, 1999). In the nuclear power HFE field, situation awareness is also recognized as an important standard for control system safety evaluation (Sebok, 2000).

The team performance evaluation techniques that greatly affect team technique research are BARS and BFRS (Behavioural Frequency Rating Scales). Such a behavior-based team performance evaluation technique emphasizes six team techniques: communication, openness, task co-ordination, team spirit, maintaining task focus and adaptability. Montgomery et al. (1991) comparatively studied BARS and BFRS as the evaluation yardstick of nuclear power plant main control room operation team. According to the study results, it was identified that BARS offer more appropriate performance standard than BFRS. BARS have strengths in such aspects as clear evaluation standard, convenience of measurement, efficient performance feedback and consistency. However, not being free from evaluation results' objectivity issue is pointed out on BARS, due to evaluator's cognitive errors and bias (Murphy, and Pardaffy, 1989; Kingstrom and Bass, 1981). The U.S. military developed and used SPOTLTE (Scenario- 
based Performance Observation Tool for Learning in Team Environments) as a team performance evaluation method to supplement BARS, instead of using BARS alone, so as to evaluate the team performance of military personnel performing tasks under the computer-based complex environment. To evaluate the team performance of Koreas' nuclear power plant main control room operators, cognitive team tasks should be effectively evaluated, and evaluation methodology that can more objectively and systematically evaluate team performance factors needs to be developed. The team performance and human performance evaluation presented above are the results of $H F E V \& V$, and has a strong aspect to be reflected only in the interface design. Therefore, an analysis through simulation data is necessary to observe actual operator's task aspects. SMART, however, acquired only standard design authorization (SDA), and there are no simulator analysis data. In this context, this study classified operator's task types by analyzing operation tasks using SMART EOG (Emergency Operating Guidance) as the underlying research to improve human performance and team performance.

\section{Case study}

As a purpose of this paper, operation tasks need to be analyzed in order to classify the operator's task types of SMART, which is a SMR. The analysis subject is SMART EOG, as presented below. Tasks were analyzed using MS Office EXCEL and Task Architect Professional 2.0. For the work to segment SMART MCR task factors, this paper analyzed them based on 10 task considerations presented by NUREG-0711 (Rev. 3) as shown in Table 1:

- Standard Post Trip Action: SPTA

- Diagnostic Actions: DA

- Reactor Trip

- Loss-of-Coolant Accident: LOCA

- Steam Generator Tube Rupture: SGTR

- Excess Steam Demand Event: ESDE

- Loss of All Feedwater: LOAF

- Loss of Offsite Power: LOOP

- Station Blackout: SBO

- Functional Recovery Guideline: FRG

Of the 10 task factors in Table 1, accurate classification of the alert, task support and hazard identification factors is difficult at the task level targeting EOG. Therefore, more accurate classification is considered to be possible, if actual operation task performance observation and experiments are possible targeting EOP (Emergency Operating Procedure) in the future. Although, the type of workload was divided into cognitive workload and physical workload, the cognitive workload was assumed to be high, considering that the analysis subject was EOG.

\section{Result}

To draw operation tasks of SMART main control room operators, this paper conducted a hierarchical task analysis (HTA), and checked detailed tasks and correlations between them. To describe task requirements, this paper devised a task analysis table and described the requirements of detailed tasks (necessary information, decision making, task response, teamwork and communication) based on HTA diagram. The task requirements analysis results according to tasks are shown in Table 2.

Based on task requirement analysis results in Table 2, task performance types by operator on the entire SMART EOG were classified. The tasks of SRO can be divided into monitoring and control information request and feedback under the high workload situation in the high emergency situation as shown in Figure 1. From the task analysis results of EOG, the decision making 
Table 1. Task consideration (NUREG-0711 Rev. 3)

\begin{tabular}{|c|c|}
\hline Topic & Example \\
\hline Alerts & - Alarms and warnings \\
\hline \multirow{2}{*}{ Information } & - Parameters (units, precision, and accuracy) \\
\hline & - Feedback needed to indicate adequacy of actions taken \\
\hline \multirow{2}{*}{ Decision-making } & - Decision type (relative, absolute, probabilistic) \\
\hline & - Evaluations to be performed \\
\hline \multirow{7}{*}{ Response } & - Actions to be taken \\
\hline & - Task frequency and required accuracy \\
\hline & - Time available and temporal constraints (task ordering) \\
\hline & - Physical position (stand, sit, squat, etc.) \\
\hline & - Biomechanics \\
\hline & - Movements (lift, push, turn, pull, crank, etc.) \\
\hline & - Forces needed \\
\hline \multirow{2}{*}{ Teamwork and communication } & - Coordination needed between the team performing the work \\
\hline & - Personnel communication for monitoring information or taking control actions \\
\hline \multirow{3}{*}{ Workload } & - Cognitive \\
\hline & - Physical \\
\hline & - Overlap of task requirements (serial vs. parallel task elements) \\
\hline \multirow{3}{*}{ Task support } & - Special and protective clothing \\
\hline & - Job aids, procedures or reference materials needed \\
\hline & - Tools and equipment needed \\
\hline \multirow{3}{*}{ Workplace factors } & - Ingress and egress paths to the worksite \\
\hline & - Workspace needed to perform the task \\
\hline & - Typical environmental conditions (such as lighting, temp, noise) \\
\hline \multirow{4}{*}{$\begin{array}{l}\text { Situational and performance } \\
\text { shaping factors }\end{array}$} & - Stress \\
\hline & - Time pressure \\
\hline & - Extreme environmental conditions \\
\hline & - Reduced staffing \\
\hline Hazard identification & - Identification of hazards involved, e.g., potential personal injury \\
\hline
\end{tabular}

and task response of SRO cannot be checked, and thus supplementation is necessary through actual operation task performance observation and experiments targeting EOP in the future.

Also, the task performance types of the two operators, RO and TO (AO), can be divided into 12 types including decision making (absolute, relative), task response (simple monitoring, continuous monitoring, simple control, continuous control, conditional control), and communication (briefing, feedback) as shown Figure 2. 
Table 2. Result of task analysis

\begin{tabular}{|c|c|c|c|c|c|c|c|c|c|c|c|c|c|c|c|c|c|c|}
\hline \multirow[t]{2}{*}{ 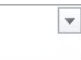 } & \multirow{2}{*}{ Strategy } & \multirow[b]{2}{*}{ Content } & \multirow[b]{2}{*}{ Strategy } & \multirow{2}{*}{$\nabla$} & \multirow[t]{2}{*}{7} & \multicolumn{2}{|c|}{ Information } & \multirow{2}{*}{$\begin{array}{c}\text { Decision } \\
\text { making }\end{array}$} & \multicolumn{2}{|c|}{ Response } & \multicolumn{2}{|c|}{ Communication $\nabla$} & \multicolumn{2}{|c|}{ Work load } & \multirow{2}{*}{$\underset{\substack{\text { Task } \\
\text { Support }}}{\text { The }}$} & \multirow{2}{*}{ Operato } & \multirow{2}{*}{ Task stress } & \multirow{2}{*}{$\begin{array}{l}\text { Hazard } \\
\text { Identification }\end{array}$} \\
\hline & & & & & & Type & Content & & \begin{tabular}{|l|} 
Frequency \\
\end{tabular} & Type & \begin{tabular}{|l|} 
personnel \\
\end{tabular} & MaI & cognitive & physical & & & & \\
\hline \multirow{4}{*}{$\begin{array}{l}\text { 4. Ensure SG } \\
\text { level is being } \\
\text { reseresed and } \\
\text { maintained }\end{array}$} & \multirow{4}{*}{ 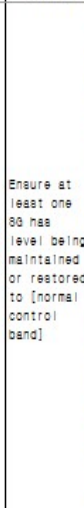 } & 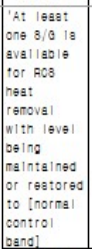 & & & & Monitoring & $\mathrm{sl}$ & Relative & Low & $\begin{array}{c}\text { Simple } \\
\text { confirmation }\end{array}$ & $\begin{array}{c}\text { Information } \\
\text { request } \\
\text { / Briefing } \\
\text { \& Feedback }\end{array}$ & Monitoring & Norm & Low & NA & AO & Low & NA \\
\hline & & 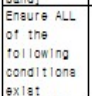 & & & & Monitoring & sl & Relative & Low & $\begin{array}{c}\text { Simple } \\
\text { confirmation }\end{array}$ & \begin{tabular}{|c|} 
Information \\
request \\
/ Brinfing \\
\& Feedback
\end{tabular} & Monitoring & Norm & Low & NA & AO & Low & NA \\
\hline & & 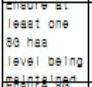 & & & & Monitoring & sl & Relative & Low & $\begin{array}{c}\begin{array}{c}\text { Simple } \\
\text { confirmation }\end{array} \\
\end{array}$ & \begin{tabular}{|c|} 
Control \\
request \\
/ Briefing \\
\& Feedback \\
\end{tabular} & Monitoring & Norm & Low & NA & AO & Low & NA \\
\hline & & $\begin{array}{l}l \\
\text { level la } \\
\text { ba ing } \\
\text { reservad } \\
\text { sna }\end{array}$ & & & & Monitoring & $\mathrm{sl}$ & Relative & Low & $\begin{array}{c}\text { Simple } \\
\text { confirmation }\end{array}$ & $\begin{array}{c}\text { Control } \\
\text { request } \\
\text { / Briefing } \\
\text { Q Feedback }\end{array}$ & Monitoring & Norm & Low & NA & A0 & Low & NA \\
\hline
\end{tabular}

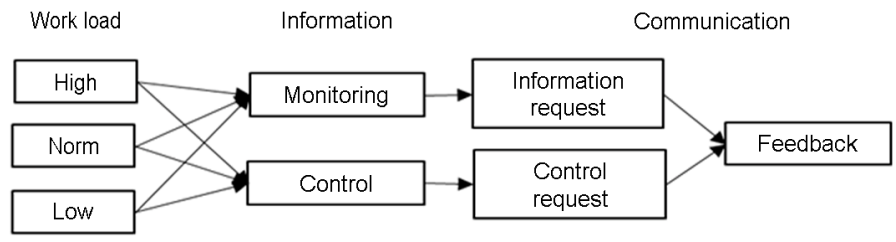

Figure 1. Type of SRO's task

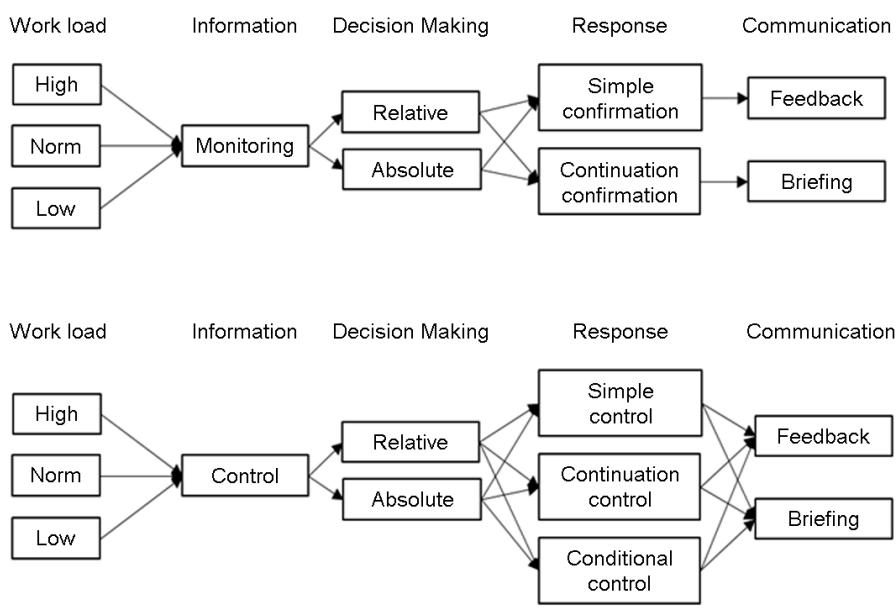

Figure 2. Type of tasks of RO and TO (AO)

From the results of task performance type classification by operator on the entire SMART EOGs, it was found that LOCA EOG had the most tasks as shown in Figure 3.

In case 10 EOGs are conducted as shown in Figure 4, RO's tasks are 69\%, the highest of the total. This implies that the role of 
ROs is huge in terms of team performance to achieve common goal of safe power plant operation. However, this result was analyzed by an analyst, based on $10 \mathrm{EOGs}$, and thus, the result may be different from actual operation results. For this reason, the result needs supplementation and correction through SMART simulation in the future. Not only the performance of the team frequently changes, but human performance by operator needs to be evaluated so that team performance can be improved by removing the factors that hinder team performance.

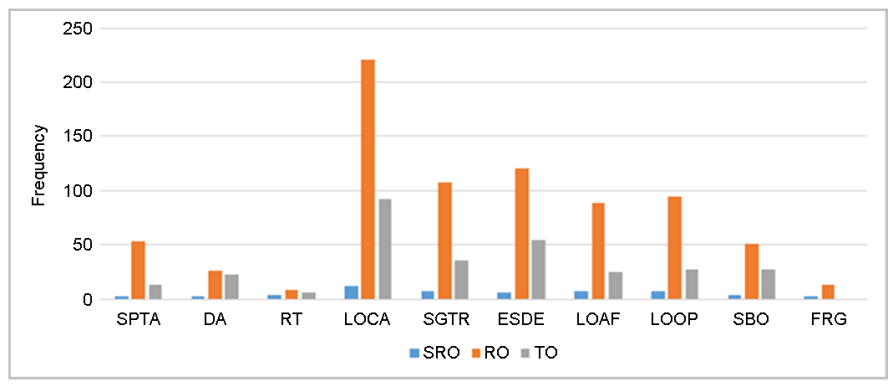

Figure 3. Frequency of operator's task

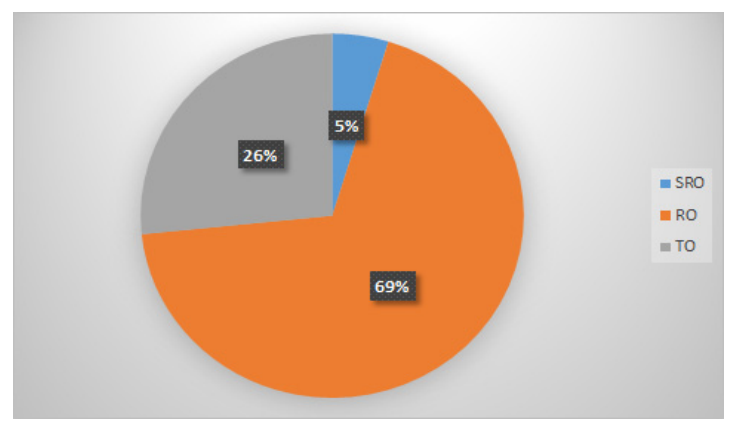

Figure 4. Ratio of operators' tasks

Especially, "absolute decision making-simple control-control request and feedback-briefing and feedback," and "absolute decision making-simple monitoring-information request and feedback-briefing and feedback" took place the most to RO and TO (AO) out of 12 task types.

Absolute decision making is a behavior to judge true or false on quantitative values, and it is the task that can be conducted with only the information concerned. Meanwhile, relative decision making is the behavior to judge by comparing with and analyzing other information in order to decide the information concerned, and is carried out to tell information change trend or discern comparative advantage. Simple control and simple monitoring are the tasks for one-off monitoring and control to operate or monitoring specific equipment or system. Continuous control and continuous monitoring are to find any change or special things of the information concerned with continuous check and monitoring or control. Conditional action is the task to operate equipment or system in line with a specific condition.

\section{Discussion/Conclusion}

This study analyzed task frequencies and types by operator targeting SMART EOG to evaluate the appropriate composition of 


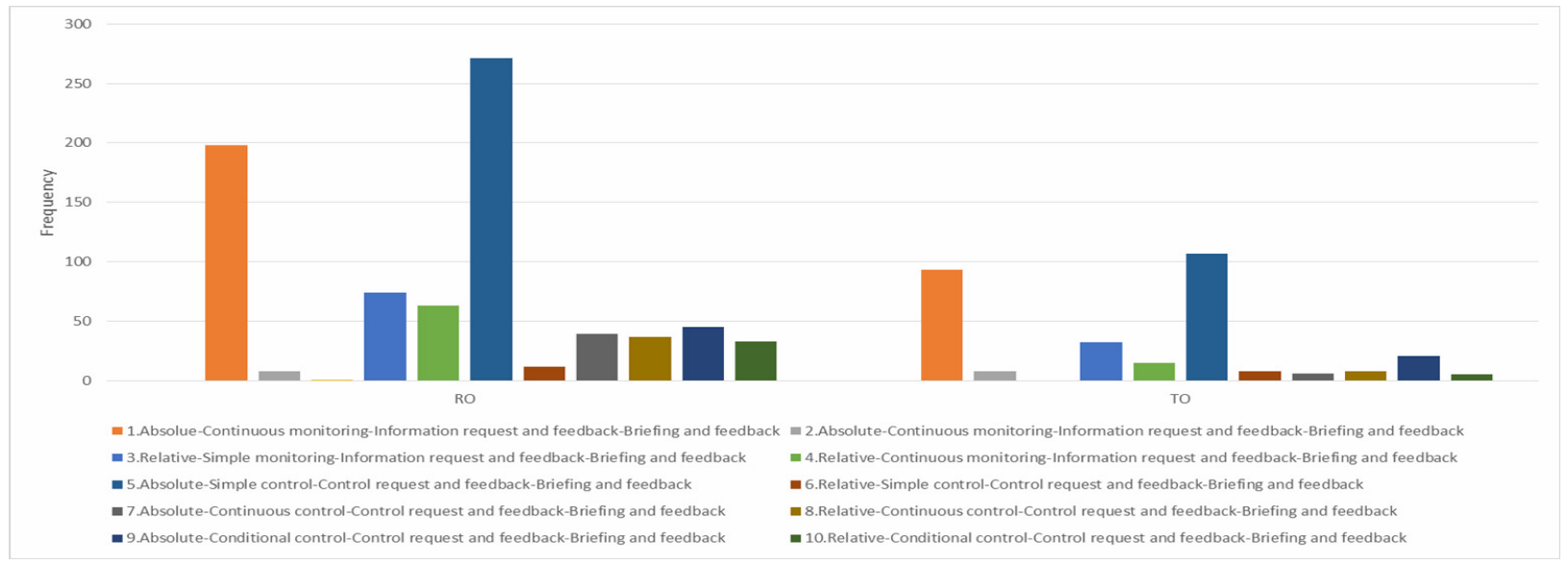

Figure 5. Types of operators' tasks

SMART operation team for the improvement of human performance and team performance. However, further study is necessary, for example on the comparison and verification results of situation awareness, mental workload and team interaction under the NUREG/IA-0137's assumption that cutting-edge control room operators are composed of two minimum. The NUREG/IA-0137 showed higher situation awareness and team interaction than conventional control room composed of four operators. In case operation is conducted with an operation team composed of two operators in the cutting-edge control room, mental workload of all the supervisors was higher. And the results actually brought up a problem. The results were from the research that just targeted the operation team. According to 12 task types, which are the results of this study, if human reliability assessment (HRA) by operator is conducted, human errors by task type are drawn, and an effort to reduce human errors by task type through HRA by operator is made, and human performance and team performance are judged to be improved furthermore. Especially, as for the "absolute decision making-simple control-control request and feedback-briefing and feedback," and "absolute decisionsimple monitoring-information request and feed back- briefing and feedback," which are task types conducted by RO and TO (AO) the most out of 12 task types, if human error type classification and mechanism are drawn up above all, and the errors are reduced, the efficiency of two-operators team composition, as well as the operation safety guarantee of RO and TO (AO) will be proved. This study has a limitation in that the human errors and SMART HRA of the operators were not drawn. This is because the limitation demonstrated when the HRA techniques that evaluated a human error possibility on the existing design are applied to improved design.

In this regard, a new technique that can analyze human errors is necessary. Thus, a technique that can take into account a new type of performance error occurrence possibility by a totally different interaction mode is demanded. Unlike the main control room in the analogue mode in the past, the role and weight of SRO relatively increases in view of the system and organizational culture, when it comes to the cutting-edge control room. This involves a possibility of difference occurring in the performance of each operator and in workload, according to SRO operation mode and knowledge including each SRO's leadership, team situation awareness and communication mode between team members. However, from the results of task analysis targeting EOG in this study, SRO's task weight is low at 5\%. This needs to be supplemented in the future through SMART HFE V\&V. Despite various limitations, the purpose of this study is basic research to set the number of operators to adjust operator's work, and thus, this study classified the task types of RO and TO (AO). Based on this, if the role and weight of SRO and task types of each operator are reevaluated by applying EOP in the future, the reevaluation results can be used as the basis to decide the appropriate number of operators of the SMR. 


\section{Acknowledgements}

This work was supported by the National Research Foundation of Korea (NRF) grant funded by the Korea government (MEST) (No. 2013M2B9A1025900).

\section{References}

Byun, S.N. and Lee, D.H., Preliminary Safety Review on the Design of Korea Next Generation Reactor: A Human Factors Evaluation of Advanced Control Facilities in Korea Next Generation Reactor, Korea Institute of Nuclear Safety, KINS/HR-404, 2001.

Coblentz, A. and Mollard, R., Human efficiency variability in monotonous conditions effects on safety, Human decision making and manual control, 76-86, 1989.

Endsley, M.R., Measurement of situational awareness in dynamic systems, Human Factors, 37, 65-84, 1995b.

Fowlkes, J.E., Lane, N.E., Dwyer, D.J., Willis, R.P. and Oser, R., Team performance measurement issues in DIS-based training environments. In Proceedings of the 14th Interservice/Industry Training Systems and Education Conference (pp.272-280). Arlington, VA: American Defense Preparedness Association, 1995.

Ha, J.S., Seong, P.H, Lee, M.S. and Hong, J.H., Development of human performance measure for human factors validation in the advanced MCR of APR-1400, IEEE Transaction on Nuclear Science, Vol.54, No.6, pp.2687-2700, 2007.

Hackman, J.R.(ed.), Groups that work (and those that Don't): Creating Conditions for Effective Teamwork, San Fransisco, CA: Jossey Bass, 1990.

Hackman, J.R., Effects of task characteristics on group products, Journal of Experimental Social Psychology, Vol.4, pp.162-187, 1968.

Hackman, J.R., A normative model of work team effectiveness (Tech. Rep. No.2), New Haven, CT: Yale University, 1983.

Hackman, J.R., Leading teams: Setting the stage for great performances, Boston: HBS Press, 2002.

Hackman, J.R. and Morris, C.G., Group tasks, group interaction process, and group performance effectiveness: A review and proposed integration, In L. Berkowitz (Ed.), Advances in experimental social psychology, Vol.8, pp.45-99, New York: Academic Press, 1975.

Helmreich, R.L., Butler, R.E., Taggart, W.R. and Wilhelm, J.A., Behavioral markers in accidents and incidents: Reference list. NASA/ UT/FAA Technical Report 95-1. Austin, TX: The University of Texas, 1995.

INPO., Control Room Teamwork Development Training: Course Administration and Facilitation Guide, National Academy for Nuclear Training, Atlanta, GA, 1993.

INSAG; International Nuclear Safety Advisory Group, Summary report on the Post-Accident Review Meeting on Chernobyl Accident. Vienna, Austria: International Atomic Energy Agency, 1986.

Kim, S.K., Development and Evaluation of Crew Resource Management Training for Improving Team Performance of Operators 
in the APR-1400 Nuclear Power Plant, Graduate School of Kyung Hee University, Dissertation of Industrial Engineering, 2008.

KINS, Development of Evaluation Techniques for Emerging Digital Application's Safety and MCR Operator's Team Performance, Korea Institute of Nuclear Safety, KINS/RR-555, 2008.

KOPEC, Engineering Report: 2th HFE V\&V Report, SNK 3\&4, Korea Power Engineering Company, 2007.

Lee, D.H., Byun, S.N. and Lee, Y.H., Short-Term Human Factors Engineering Measures for Minimizing Human Error in Nuclear Power Facilities, Journal of the Ergonomics Society of Korea, 26(4), 121-125, 2007.

Montgomery, J. Gaddy C. and Toquam, J., Team interaction skills evaluation criteria for nuclear power plant control room operators, Proceedings of the Human Factors Society 35th Annual Meeting, 2-6September, 918-22, Santa Monica, CA: HFS, 1991.

Mumaw, R., Swatzler, D., Roth, E. and Thomas, W., Cognitive skill training for nuclear power plant operational decision making (NUREG/CR-6126), Washington, DC: U.S. Nuclear Regulatory Commission, 1994.

Norman, D.A. and Bobrow, D.G. On data-limited and resource-limited processes. Cognitive Psychology, 7, 44-64, 1975.

O'Donnell, R.D. and Eggemeier, F.T., Workload assessment methodology. In K. R. Boff, L. Kaufman, and J. Thomas, Eds., Handbook of Perception and Human Performance; volumeII, cognitive Processes and Performance. New York, NY: J. Wiley and Sons, 1986.

O'Hara, J.M. and Hall, R.E., Advanced control rooms and crew performance issues: implications for human trust, Brookhaven National Laboratory, NewYork 11973, 1405-1409, 1990.

Rasmussen, J., Information processing and human-machine interaction, Amsterdam. North Holland, 1986.

Reason, J., The Chernobyl errors. Bulletin of the British Psychological Society, 40, 201-206, 1987.

Rogovin, M., Grampton, G.T. and the Nuclear Regulatory Commission (NRC) Special Inquiry Group, Three Mile Island: $A$ report to the Commission and the public (NUREG/CR-1250, Vol. 1). Washington, DC: U.S. Nuclear Regulatory Commission 1980.

Roth, E.M. et al., An empirical investigation of operator performance in cognitively demanding simulated emergencies, NUREG/ CR-6208. Washington, DC: USNRC, 1994.

Salas, E. and Cannon-Bowers, J.A., The science of training: A decade of progress, Annual Review of Psychology, 52, 471-499, 2001.

Seamster, T.L. et al., Developing Advanced Crew Resource Management (ACRM) Training: A Taining Manual 1998.

Sebok, A., Team performance in process control: influences of interface design and staffing levels, Ergonomics, 43(8), 1210-1236, 2000.

Stout, R.J., Cannon-Bowers, J.A. and Salas, E., The role of shared mental models in developing team situational awareness: Implications for training. Training Research Journal, 2, 85-116, 1996.

Stout, R.J., Salas, E. and Fowlkes, J., Enhancing teamwork in complex environments through team training. Group Dynamics: Theory, 
Research, \& Practice, I, 169-182, 1997.

Tannenbaum, S.I. and Yukl, G., Training and development in work organization, Annual Review of Psychology, 43, 339-441, 1992.

United States Congress, Section 306 of the Nuclear Waste Policy Act of 1982 (Public Law 97-425) 1982.

USNRC, NRC action plan developed as a result of the TMI-2 Accident (NUREG-0660), Washington DC: US Nuclear Regulatory Commission, 1980.

USNRC, Operator licensing examiner standards (NUREG-1021), Washington DC: US Nuclear Regulatory Commission, 1989.

USNRC(?), A Study of Control Room Staffing Levels for Advanced Reactors(NUREG/IA-0137) Washington DC: US Nuclear Regulatory Commission.

Wigdor, A.K. and Green, B.F., Jr., Performance assessment for the workplace. Washington, DC: National Academy Press, 1991.

Wickens, C.D., Engineering Psychology and Human Performance. New York, NY; Harper Collins, 1992.

Wickens, C.D., Gordon, S.E. and Liu, Y., An Introduction to Human Factors Engineering. New York, NY: Longman, 1998.

Williges, R. and Wierwille, W.W., Behavioral measures of aircrew mental workload. Human Factors, 21, 549-574, 1979.

\section{Author listings}

Eun Mee Heo: hem2040@khu.ac.kr

Highest degree: MS, Department of International Management, Kyunghee University

Position title: Ph.D. candidate, Department of Industrial Engineering, Kyunghee University of Yong-in

Areas of interest: Human Factors in Nuclear Power Plant, HRA, HCI, Team Performance

Seong Nam Byun: snbyun@khu.ac.kr

Highest degree: Ph.D. Department of Industrial Engineering, The University of Michigan

Position title: Professor, Department of Industrial \& Management Engineering, Kyunghee University of Yong-in

Areas of interest: Human Factors in Nuclear Power Plant, Training, HCI, HRA

Hong Joon Park: groovyhong@khu.ac.kr

Highest degree: MS, Department of Industrial Engineering, Kyunghee University

Position title: Ph.D. candidate, Department of Industrial Engineering, Kyunghee University

Areas of interest: Human Factors in Nuclear Power Plant, Training, HCI

Geun Ok Park: gopark@kaeri.re.kr

Highest degree: Ph.D. Department of Computer Engineering, Kongju National University

Position title: Project Manager, Department of Research Reactor Engineering, Korea Atomic Energy Research Institute

Areas of interest: Human Factors in Nuclear Power Plant, Training System in Nuclear Industry, Simulator, HMI 